\title{
Staged Hybrid Endovascular Repair of a Ruptured Abdominal Aortic Aneurysm with Aortocaval Fistula
}

\author{
Sandra A Chung, BA ${ }^{1}$, Chris M Reid, $\mathrm{MD}^{2}$, Dennis F Bandyk, MD³ , Andrew Barleben, MD MPH ${ }^{3 *}$, \\ John S Lane III, MD ${ }^{3}$ \\ ${ }^{1}$ School of Medicine, University of California San Diego, La Jolla, California, USA \\ ${ }^{2}$ Department of Surgery, University of California San Diego Medical Center, San Diego, California, USA \\ ${ }^{3}$ Division of Vascular and Endovascular Surgery, University of California San Diego Medical Center, San Diego, California, USA
}

\begin{abstract}
There is a growing body of literature expanding the indication of endovascular aneurysm repair, from prophylactic treatment of aneurysms to other indications such as ruptured and complicated ruptured abdominal aneurysms. Concomitant aortocaval fistula is rare, and reports of open and endovascular repair exist. We report a unique hybrid approach to a case of a ruptured abdominal aortic aneurysm with aortocaval fistula, repaired primarily via endovascular approach in a hybrid, two-staged fashion. Representative images are presented in addition to a short review of this pathology.

Copyright $\odot 2015$ Science International Corp.
\end{abstract}

\section{Key Words}

Ruptured abdominal aortic aneurysm - Aortocaval fistula • EVAR

\section{Introduction}

Spontaneous aortocaval fistula (ACF) is a rare complication of abdominal aortic aneurysm (AAA), present in fewer than $1 \%$ of all AAAs and up to $6 \%$ of ruptured AAAs [1]. In addition to the classic triad of hypotension, flank/back pain, and a pulsatile abdominal mass seen in ruptured AAAs, the clinical presentation of a ruptured AAA with ACF may include

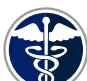

Fax +1 2037853552

E-Mail: aorta@scienceinternational.org

http://aorta.scienceinternational.org

\author{
(c) 2015 AORTA \\ Published by Science International Corp. \\ ISSN 2325-4637 \\ Accessible online at: \\ http://aorta.scienceinternational.org
}

a continuous machinery murmur, lower extremity swelling, and high-output cardiac failure [1]. Conventional open repair of a ruptured AAA has a high mortality rate and is decreasing in frequency [2]. In select patients, the preferred treatment for ruptured AAAs and ruptured AAA complicated by ACF is endovascular aneurysm repair (EVAR), which avoids the morbidity of open repair [3]. However, data are conflicting on whether there is a significant survival benefit of EVAR over an open approach in the repair of ruptured AAA [2-4]. A review of the literature reveals there is scant data on the management of ruptured AAA presenting with ACF. We report a case of ruptured AAA with ACF successfully repaired via a two-stage hybrid approach.

\section{Case Report}

An 85-year old man with a 72 pack-year smoking history, but otherwise no significant past medical history, was transferred to our hospital for emergent repair of a ruptured AAA after presenting to another hospital with a 5-day history of lower back pain and one day of hematemesis. Prior to presentation, he had been in good health and denied symptoms of peripheral venous hypertension. Computed tomography angiography (CTA) showed a 9.2-cm AAA with

\footnotetext{
* Corresponding author:

Andrew Barleben, MD MPH

Department of Surgery

University of California San Diego, Division of Vascular and Endovascular Surgery

Mail Code 7403, 9434 Medical Center Drive, La Jolla CA 92037-1300

Tel.: +1 858657 8530, Fax: +1 858657 5033, E-Mail: abarleben@ucsd.edu
} 

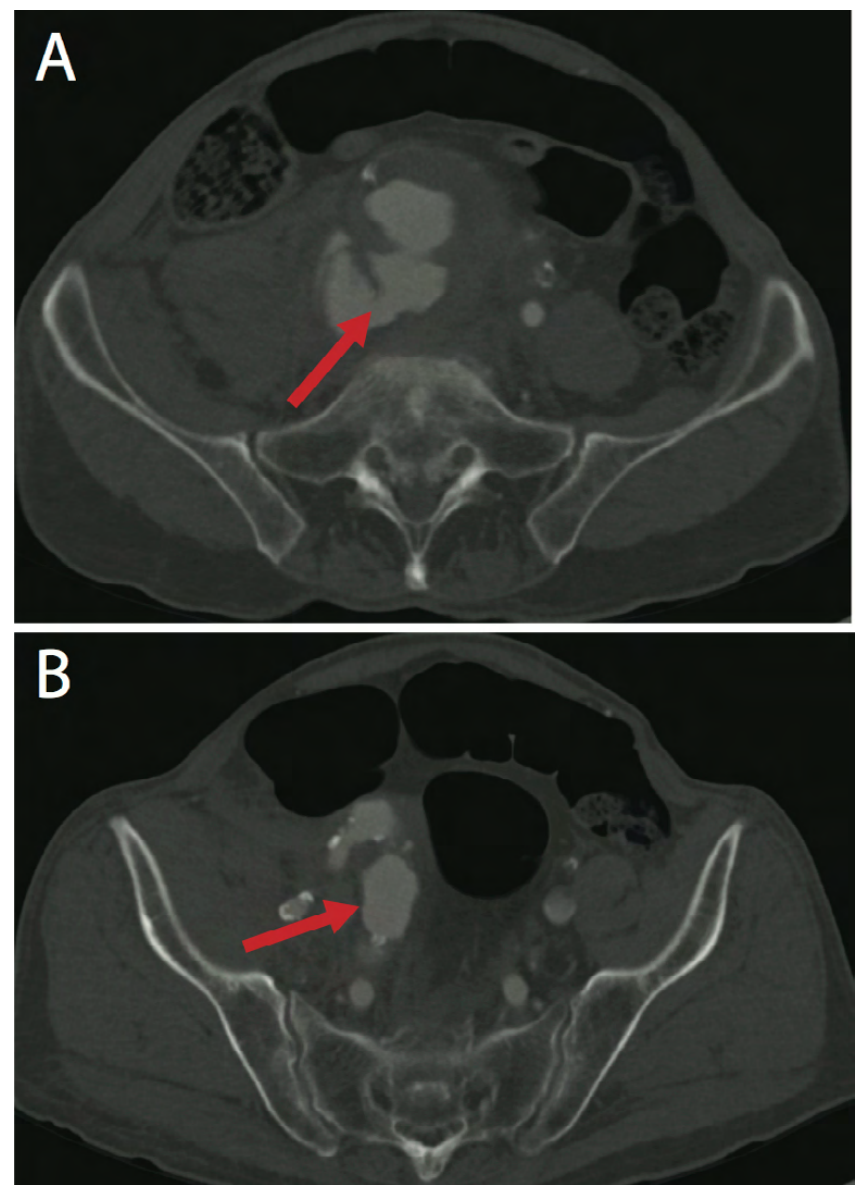

Figure 1. A. Initial computed tomography angiogram showing ruptured AAA into aortocaval fistula (arrow). B. Hypogastric artery aneurysm (arrow).

extensive rupture into the retroperitoneum, a right hypogastric artery aneurysm with iliocaval fistula, a duplicated caval system, and an occluded left iliac artery (Figure 1). On exam, the patient was hypotensive and had a soft, minimally distended abdomen. Given the patient's clinical status and features of the AAA, the decision was made to proceed to the operating room for endovascular repair. Intraoperative ultrasound also showed a right common femoral artery aneurysm and occlusion of the left common femoral artery. Subsequently, a femoral cutdown was performed on the right groin. Planned coverage of the right hypogastric artery was executed due to the fact that the iliocaval fistula was emanating from the right hypogastric vessel. Dual C3 GORE Excluder main body bifurcated devices (Flagstaff, Arizona, USA) were deployed with exclusion of the right hypogastric artery in an aorto-uni-iliac fashion. Hemodynamic changes consistent with coverage of an arteriovenous fistula were observed, including a rise in blood pressure and a decrease in cardiac output as measured on transesophageal echocardiogram. Completion intraoperative angiogram showed complete exclusion without evidence of obvious endoleak and obliteration of the iliocaval fistula. The right common femoral aneurysm was then repaired and a right-to-left femoral-femoral bypass was performed.

One week following the initial operation, the patient developed symptoms suggestive of high-output cardiac failure. Follow-up CTA showed a type II endoleak (T2E) with continued communication between the aneurysmal right hypogastric artery and the inferior vena cava (Figure 2). The patient was taken back to the operating room for open ligation of the right hypogastric artery aneurysm and inferior mesenteric artery. Once the aneurysm was excluded, it was interrogated with intraoperative ultrasound. There was continued pulsatility within the aneurysm with no flow detected in the sac on ultrasound. Flow patterns in the vena cava also demonstrated normal venous phasic flow. Repeat CTA one week later demonstrated patency of the graft and completed exclusion of the endoleak (Figure 3). Postoperative course was uneventful and the patient was discharged from the hospital in improved condition. At the 9-month follow-up, he was in good health with repeat CTA re-demonstrating patency of the graft without evidence of rupture or leak, as well as decrease in the size of the AAA.

\section{Discussion}

Ruptured AAA is a potentially lethal condition for which emergent surgical intervention is required. The addition of the rare complication of ACF presents a particularly unique treatment challenge. In those patients with ruptured AAA whose anatomy is suitable for endovascular repair, EVAR may confer a lower operative mortality and perioperative morbidity than conventional open repair [3]. However, data is conflicting on whether this early benefit persists beyond the operative and perioperative period [2-4]. Data are even more limited in cases of ruptured AAA with the rare complication of ACF. 


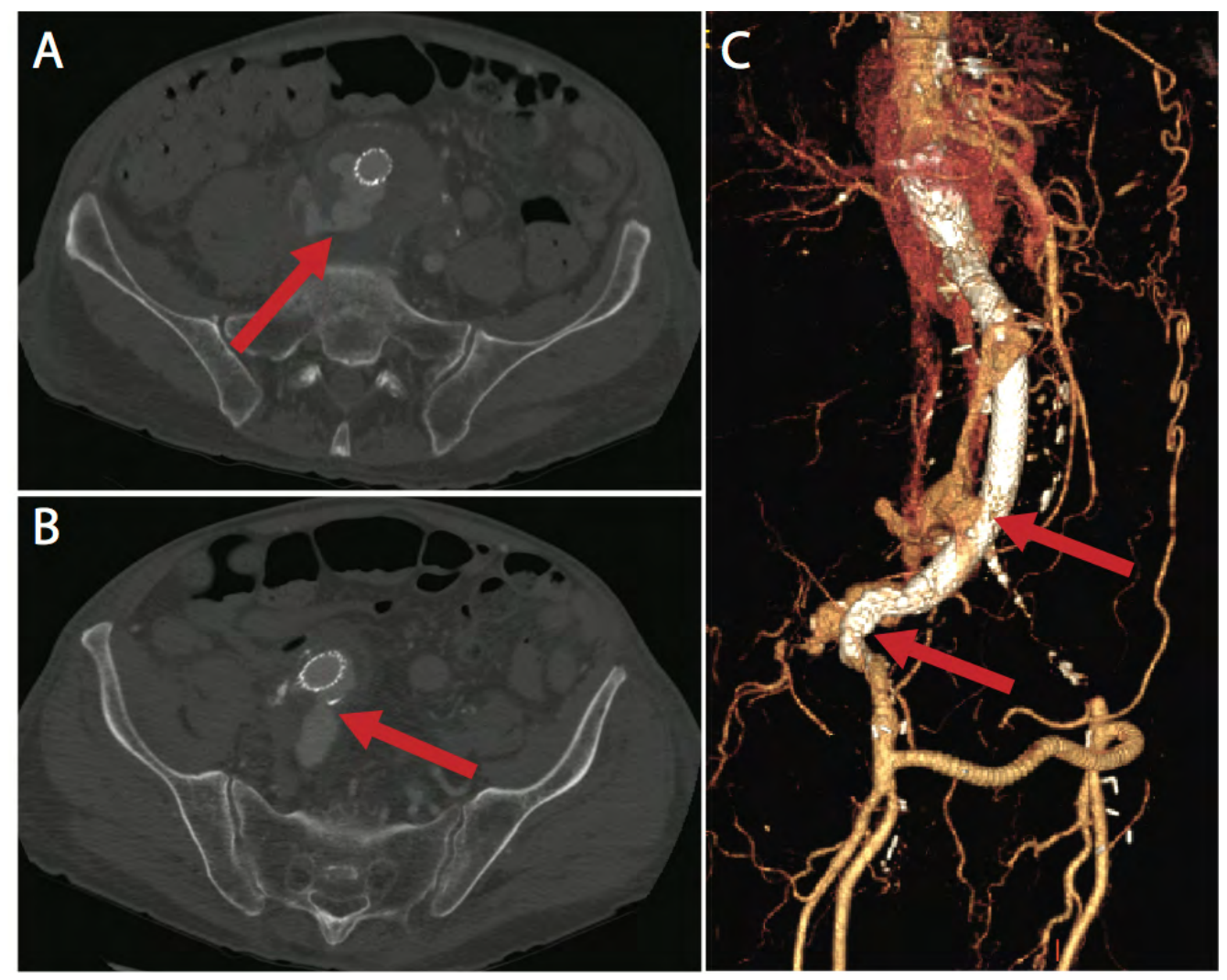

Figure 2. A. Computed tomography angiogram showing persistent aortocaval fistula (arrow). B. Type II endoleak from backfilling aneurysmal hypogastric artery (arrow). C. Three-dimensional reconstruction with contrast filling around the graft (arrows).

Additionally, in the emergent management of this life-threatening condition, treatment decisions must be tailored to the unique aspects of the case. In this case, once the orifice to the internal iliac artery was covered in the first stage, we no longer had access to the vessel using endovascular techniques. However, it is our standard of practice at our institution to plug or coil-embolize internal iliac arteries or perform iliac-preserving techniques when extension is needed into the external iliac artery. Secondly, due to immediate availability, Dual GORE Excluder devices were used rather than an off-the-shelf aorto-uni-iliac device from another company. In the second stage, an open transabdominal rather than retroperitoneal approach was taken given the relative complexities of the patient's anatomy: The large internal iliac artery aneurysm and the desires for the ability to have proximal control and to analyze the aneurysm sac using direct duplex ultrasound.
In the presence of ACF, endovascular repair is accompanied by the theoretical concern of high flow T2Es, which could lead to persistent aortocaval communication. T2Es occur after EVAR and are caused by retrograde flow of collateral arteries into the excluded aneurysm sac, leading to repressurization and consequent aneurysm growth and, ultimately, possible rupture [5]. Without surgical intervention, a T2E in the setting of persistent aortocaval communication may lead to high-output cardiac failure and sac growth. In many cases, the flow of a T2E may be small enough as to not lead to sac enlargement of a continuous ACF. However, in the setting of a rupture, the integrity of the wall of the aorta is already compromised, and there is an increased risk of progression in the presence of both a T2E and venous fistula. Although the role of intervention in patients with T2Es remains controversial in the literature, there is consensus that this pathology is accompanied with high risks of morbidity and mortality. 

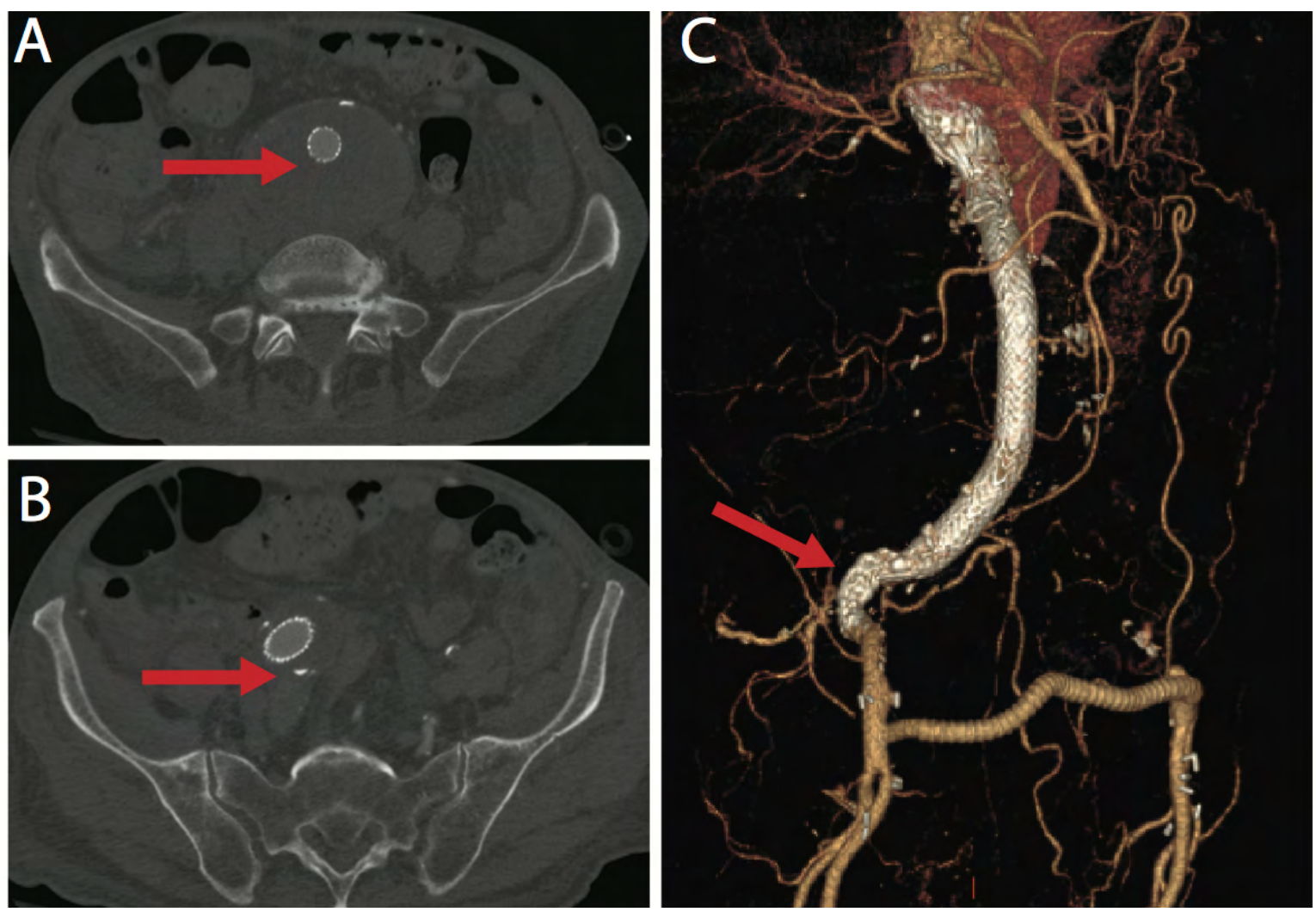

Figure 3. A. Computed tomography angiogram showing resolution of aortocaval fistula (arrow). B. Resolution of type II endoleak (arrow). C. Three-dimensional reconstruction with resolution of endoleak (arrow) and patent bilateral runoff.

Several case reports and case series describe successful EVAR or open surgical repair of unruptured AAAs with ACF $[5,6]$ and many cases can be found of AAA with contained ruptures into the inferior vena cava (IVC) repaired via both endovascular [7] and open approaches [1]. However, reports of AAA complicated by both a free rupture into the retroperitonium and aortocaval fistulization are extremely rare. Melas et al. [8] reported their repair of a leaking AAA via EVAR with an aorto-uni-iliac stent-graft followed by the late detection of a T2E and ACF, which was treated with an IVC endograft. Unosawa et al. [9] repaired a ruptured AAA with a non-bleeding, occluded ACF through an open approach. We found only one case report, by Psathas et al. [10], in which an AAA was found with a coexistent retroperitoneal rupture and free-flowing ACF which was repaired with open surgery.

We report the successful treatment of a freely ruptured AAA complicated by ACF via a unique staged, hybrid approach. In the first stage, hemodynamic stability was achieved with endovascular exclusion of the AAA and exclusion of an aortocaval fistula with an aortic-uni-iliac stent graft. In the second stage, an open repair of a T2E allowing persistent arteriovenous communication was performed without the high risks associated with open repair of ruptured AAA and associated ACF.

The literature on AAA with ACF is sparse, limited to case studies, and those patients presenting in the emergent setting of a freely ruptured AAA with ACF are rarer still. The paucity of data hinders the comparison of outcomes of open versus endovascular repair of this rare and complicated condition. The emergent management of these cases, therefore, presents a unique and difficult challenge. As described above, endovascular placement of a stent graft can potentially act as an initial damage control therapy in the setting of a life-threatening ruptured AAA and may create a role for the staged repair of concomitant, but not immediately life-threatening lesions, such as ac- 
cessory aneursyms or ACF. This hybrid, staged strategy may prove to be the preferred approach for select patients presenting with this rare complication. Further study is necessary to better evaluate the efficacy and safety of this method.

Ruptured AAA complicated by ACF is rare and life threatening. We present the successful treatment of a freely ruptured AAA with ACF via a staged, hybrid approach. EVAR may serve as the preferred initial management approach followed by open repair of concomitant but not immediately life-threatening lesions, such as ACF.

\section{References}

1. Kotsikoris I, Papas TT, Papanas N, Maras D, Andrikopoulou M, Bessias N, et al. Aortocaval fistula formation due to ruptured abdominal aortic aneurysms: A 12-year series. Vasc Endovascular Surg. 2013 47: 5156. DOI: $10.1177 / 1538574411418842$

2. IMPROVE Trial Investigators, Powell JT, Sweeting MJ, Thompson MM, Ashleigh R, Bell R, et al. Endovascular or open repair strategy for ruptured abdominal aortic aneurysm: 30 day outcomes from IMPROVE randomised trial. BMJ. 2014;348:f7661. DOI: 10.1136/bmj.f7661

3. Antoniou GA, Georgiadis GS, Antoniou SA, Pavlidis P, Maras D, Sfyroeras GS, et al. Endovascular repair for ruptured abdominal aortic aneurysm confers an early survival benefit over open repair. J Vasc Surg. 2013;58:1091-1105. DOl: 10.1016/j. jvs.2013.07.109

4. ReimerinkJJ, Hoornweg LL, Vahl AC, Wisselink W, van den Broek TA, Legemate DA, et al. Endovascular repair versus open repair of ruptured abdominal aortic aneurysms: A multicenter randomized controlled trial. Ann Surg. 2013;258:248-256.
DOI: 10.1097/sla.0b013e31828d4b76

5. Gonçalves FB, Rouwet EV, Hendriks JM, Raa S ten, Verhagen HJM with Koen M. van de Luijtgaarden. Conservative management of persistent aortocaval fistula after endovascular aortic repair. J Vasc Surg. 2013;58:1080-1083. DOI: 10.1016/j. jvs.2012.10.138

6. Maeda H, Umezawa H, Goshima M, Hattori T, Nakamura T, Nishii T, et al. Surgery for ruptured abdominal aortic aneurysm with an aortocaval and iliac vein fistula. Surg Today. 2007;37:445-446. DOI: 10.1007/ s00595-006-3429-9

7. Shah TR, Parikh P, Borkon M, Mocharla R, Lonier J, Rosenzweig BP, et al. Endovascular repair of contained abdominal aortic aneurysm rupture with aortocaval fistula presenting with high-output heart failure. Vasc Endovascular Surg. 2013;47:51-56. DOI: $10.1177 / 1538574412462633$

8. Melas N, Saratzis A, Saratzis N, Lazaridis I, Kiskinis D. Inferior vena cava stent-graft placement to treat endoleak associated with an aortocaval fistula. J Endovasc Ther. 2011;18:250-254. DOI: 10.1583/10-3296.1
9. Unosawa $S$, Kimura H, Niino T. Surgical repair of ruptured abdominal aortic aneurysm with non-bleeding aortocaval fistula. Ann Vasc Dis. 2013;6:209-211. DOI: 10.3400/avd.cr.12.00106

10. Psathas ED, Lioudaki S, Doulaptsis M, Charalampoudis $\mathrm{P}$, Klonaris $\mathrm{C}$, Verikokos C. Clinical manifestations of aortocaval fistulas in ruptured abdominal aortic aneurysm: Report of two cases. Case Rep Surg. 2012;2012:1-4. DOI: 10.1155/2012/123081

Cite this article as: Chung SA, Reid CM, Bandyk DF, Barleben A, Lane III JS. Staged Hybrid Endovascular Repair of a Ruptured Abdominal Aortic Aneurysm with Aortocaval Fistula. AORTA 2015;3(1): 26-30. DOI: http://dx.doi. org/10.12945/j.aorta.2015.14-053 